\title{
Synthesis, Growth and Characterization of Benzophenone Added Sodium Acid Phthalate Crystal-A Potential Material for Nonlinear Optical Applications
}

\author{
Anandaraj Louis ${ }^{1,2}$, Jothi Lakshmanan ${ }^{3 *}$ \\ ${ }^{1}$ PG \& Research Department of Physics, Sacred Heart College (Autonomous), Tirupattur, Tamil Nadu, India \\ ${ }^{2}$ Periyar University, Palkalai Nagar, Salem, Tamil Nadu, India \\ ${ }^{3}$ PG \& Research Department of Physics, Namakkal Kavignar Ramalingam Government Arts College for Women, Namakkal, \\ Tamil Nadu, India \\ Email: *jothilakshmanan@gmail.com
}

How to cite this paper: Louis, A. and Lakshmanan, J. (2022) Synthesis, Growth and Characterization of Benzophenone Added Sodium Acid Phthalate Crystal-A Potential Material for Nonlinear Optical Applications. Journal of Minerals and Materials Characterization and Engineering, 10, 15-27.

https://doi.org/10.4236/jmmce.2022.101002

Received: November 26, 2021

Accepted: January 9, 2022

Published: January 12, 2022

Copyright $\odot 2022$ by author(s) and Scientific Research Publishing Inc. This work is licensed under the Creative Commons Attribution International License (CC BY 4.0).

http://creativecommons.org/licenses/by/4.0/

\section{Open Access}

\begin{abstract}
Sodium acid phthalate $\mathrm{C}_{8} \mathrm{H}_{5} \mathrm{NaO}_{4}$ is one of the suitable semiorganic single crystals hired in second harmonic generation conversion applications. In the present work, optically transparent Benzophenone doped with sodium acid phthalate (SAPB) single crystals were grown successfully by slow evaporation solution technique. Single crystal XRD analyses exposed that the crystal lattice of SAPB crystal is a monoclinic crystal system with unit cell parameters $a=$ $6.77 \AA, b=9.31 \AA, c=13.58 \AA$, and space group B2cb. The crystalline nature of SAPB material was confirmed by powder XRD pattern. The functional groups present in the grown crystals were identified by the FTIR analysis ranging between 4000 and $500 \mathrm{~cm}^{-1}$. The optical transparency and band gap of grown crystals were measured from UV-Visible spectroscopy. The fluorescence emission spectrum of the SAPB crystal having a strong Yellow emission peak at $574 \mathrm{~nm}$ and the red emission at $631 \mathrm{~nm}$ are identified. The Vickers microhardness number $\left(H_{v}\right)$ increases with increasing load. Meyer's index number ( $n)$ calculated from $H_{V}$ shows that the material belongs to the soft material category. Preliminary measurement using Kurtz powder technique with Nd-YAG laser light of wavelength $1064 \mathrm{~nm}$ indicates that their second harmonic generation (SHG) efficiencies of the grown crystals were presented 2 times in comparison with Potassium Dihydrogen Phosphate (KDP) was confirmed.
\end{abstract}

\section{Keywords}

Growth from Solution, UV-Vis, FTIR, Powder XRD, Fluorescence, SHG 


\section{Introduction}

Recent interest in research focuses much on the search for highly efficient second order nonlinear optical (NLO) materials due to their potential applications in data storage devices, optical communication, optical switches, optical frequency conversion, laser-based images, optoelectronics, telecommunication [1] [2]. The desired nonlinear optical material must exhibit large second-order optical nonlinearities, Semiorgani NLO crystals have a wide range of applications like high transparency range and the material is sodium acid phthalate $\left(\mathrm{C}_{8} \mathrm{H}_{5} \mathrm{NaO}_{4}\right)$ doping of external impurity plays a crucial role in tuning the properties of the host crystal, the most effective strategy to gain improvement in intrinsic properties of SAPB crystal is to incorporate benzophenone $\left(\mathrm{C}_{13} \mathrm{H}_{10} \mathrm{O}\right)$ impurities in a selected quantity [3] [4] [5] [6] [7]. To enhance the physical properties of single crystals impurities is playing a vital role. The fact motivates the abundant benzophenone chosen as dopant [8]. The excellent applications and use of titled material in various optical devices encourage us to grow good quality single crystals using dopant with improved optical properties for future technological application.

In this present work, we report the repercussion of benzophenone in sodium acid phthalate single crystals for SHG applications [9]. The method used for the growth of benzophenone doped sodium acid phthalate was the conventional method which can also be called as slow evaporation solution growth method. The grown crystal was subjected to various characterization studies such as the structural studies were made on the grown crystal by single crystal X-ray diffraction studies [10] [11] [12] [13] [14]. The crystalline nature and functional group were identified by powder X-ray diffraction, Fourier transform infrared analysis, UV-absorption studies, and the fluorescence study exhibits that material having a yellow color emission, Vickers microhardness, Finally, the NLO property of the SAPB crystal was studied using Kurtz powder technique.

\section{Experimental Procedure}

\section{Synthesis and Crystal Growth}

All the reagents were purchased from Sigma Aldrich and used without further purification. The title compound was synthesized by sodium hydroxide and phthalic acid at 1:1 equimolar ratio. The calculated amount of sodium hydroxide and phthalic acid was added to double distilled water according to the solubility. The benzophenone was dissolved in deionized water according to the solubility and stirred for 2 hour, and finally, both solutions was mixed with continuous stirring for about 3 hour using magnetic stirrer and to obtain a homogenous mixture. The completely dissolved solution was filtered using whattman filter paper to remove the suspended impurities and allowed to crystallize by solution growth method at room temperature for about 16 days. Finally a well-defined single crystal with the dimensions of $5 \times 6 \times 2 \mathrm{~mm}^{3}$ was obtained. The photograph of grown SAPB crystal is shown in Figure 1 . 


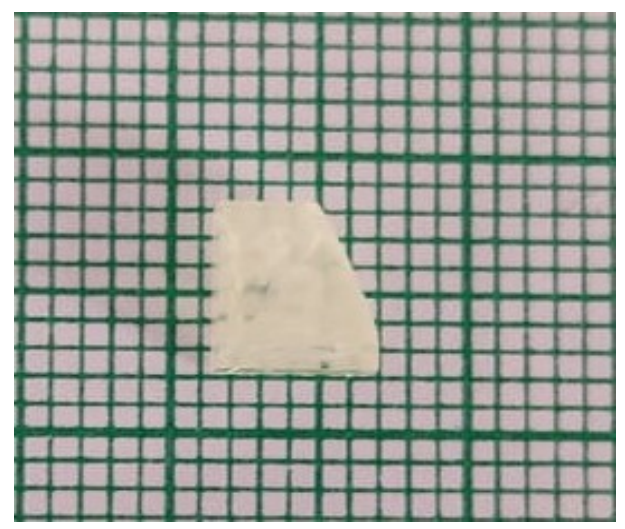

Figure 1. Photograph of as grown SAPB crystal.

\section{Result and Discussion}

\subsection{Powder X-Ray Diffraction}

The powder X-ray diffraction analysis of SAPB was recorded by using a powder $\mathrm{X}$-ray diffractometer with $\mathrm{CuKa}_{1}$ radiation $(\lambda=1.5460 \mathrm{~m})$. Figure 2 shows the powder X-ray diffraction pattern that confirms the crystalline nature of the grown crystal [15] [16] [17] [18]. From the spectrum, it is observed that the peaks are turned up in between 10' - 60' degrees. From the above investigation, we can propose that the grown crystal is a combination of reflections from (110), (011), (020), (111), (210) and (131) diffraction planes, which appeared at the $2 \theta$ of $16.54,19.06,22.28,23.38,28.52$ and 39.79 respectively, the sharp and prominent peak at 19.06 is assigned to the (001) crystal plane of SAPB. The high intensity and low full width half maxima of the peaks confirm the good crystalline nature of the crystal.

\subsection{Single Crystal X-Ray Diffraction}

The single crystal XRD studies are carried out with more dependable information on lattice parameters as compared to the powder XRD studies. The apt size of a single crystal was selected for the XRD analysis and to evaluate the cell parameters [19]. The SCXRD result declares that benzophenone doped with sodium acid phthalate crystal belongs to the monoclinic crystal system [20]. The unit cell dimensions and X-ray intensity data of SAPB were founded using Bruker D8 Venture Scxrd equipped with MoK $\alpha$ radiation $(\lambda=0.71073 \AA)$. The single crystal XRD result revealed that lithium benzophenone doped Sodium acid phthalate crystal belongs to the monoclinic system with a space group of B2cb having non-centro symmetry. The obtained lattice parameters are shown in Table 1.

\subsection{FTIR Analysis}

FTIR spectroscopy is an effective tool to identify and confirm a compound by analyzing the functional groups present within the spectrum. The molecular interactions between the reagents can be well explained by examining different 
modes of vibrations [21]-[28]. The spectrum of synthesized material, SAPB is recorded in the region of 4000 and $500 \mathrm{~cm}^{-1}$ and is depicted in Figure 3. The frequency of $350.10 \mathrm{~cm}^{-1}$ is due to $\mathrm{OH}$ overtones of the water molecule. The characteristic stretching vibrations of $\mathrm{CH}_{2}$ are observed at $2854.21 \mathrm{~cm}^{-1}$ and $2454.23 \mathrm{~cm}^{-1}$. The peaks obtained at $1598.42 \mathrm{~cm}^{-1}$ and $1626.01 \mathrm{~cm}^{-1}$ are due to $\mathrm{C}=\mathrm{O}$ stretching of the $\mathrm{COO}$ group. The aromatic ring structure of the SAPB occurs at $1571.15 \mathrm{~cm}^{-1}$ and aromatic amine stretching at $1297.06 \mathrm{~cm}^{-1}$. The $\mathrm{NH}$ bonding vibration occurs at $1626.01 \mathrm{~cm}^{-1}$. The skeletal vibrations of the aromatic ring of benzophenone were observed at $1598.42 \mathrm{~cm}^{-1}$ and $1571.15 \mathrm{~cm}^{-1}$. The vibrations proved the presence of expected functional groups in the synthesized compound.

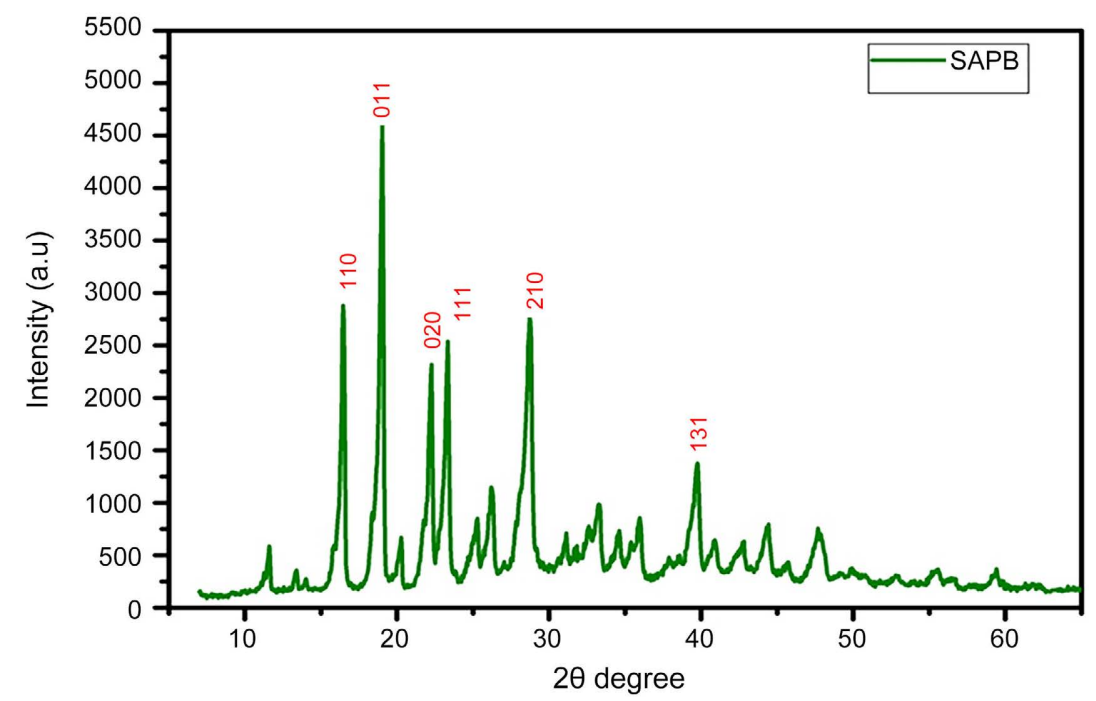

Figure 2. Powder X-ray diffraction pattern of SAPB crystal.

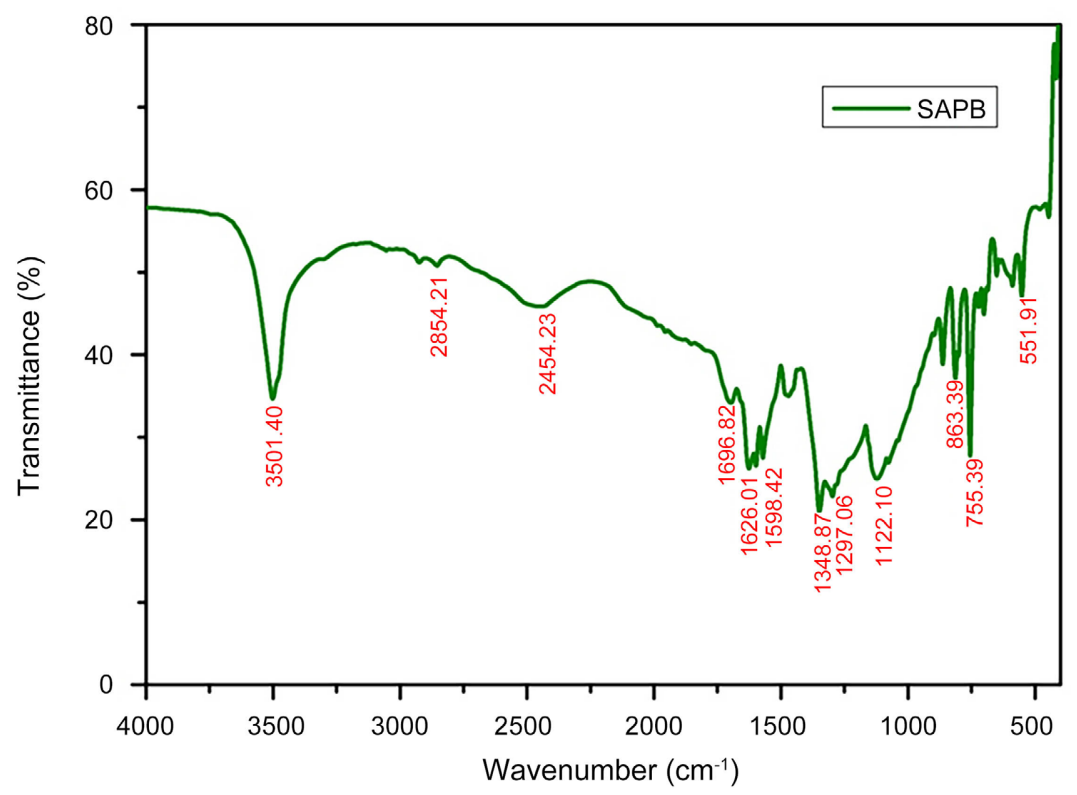

Figure 3. FT-IR spectrum of SAPB crystal. 
Table 1. Single crystal data of SAPB grown crystal.

\begin{tabular}{cc}
\hline Lattice Parameters & SAPB \\
\hline$a$ & $6.77 \AA$ \\
$c$ & $9.31 \AA$ \\
$\alpha$ & $13.58 \AA$ \\
$\beta$ & $90.05^{\circ}$ \\
$\gamma$ & $104.34^{\circ}$ \\
Volume & $90.04^{\circ}$ \\
Crystal system & $829 \AA^{3}$ \\
Space group & Monoclinic \\
\hline
\end{tabular}

\subsection{UV-VIS NIR Spectral Analysis}

The grown crystal of benzophenone doped sodium acid phthalate dissolved in distilled water was subjected to spectral studies within 200 - $1200 \mathrm{~nm}$ wavelength range using Varian Cary 5E-UV spectrometer. The optical transmittance range and lower cut-off wavelength of a single crystal are vital factors for optical applications. The obtained optical transmittance and absorption spectrum are shown in Figure 4(a) and Figure 4(b). The absorption and transmission spectra help us to identify the nature of the molecule by analyzing the transition of electrons in $\sigma$ and $\pi$ orbital's between the ground state and higher excited states with the energy absorbed [29] [30]. The SAPB single crystal showed 93\% in the entire visible region with the cut-off wavelength of $277 \mathrm{~nm}$. The spectrum indicates that the crystal to be used as a promising aspirant for a higher order of non-linear optical generations and terahertz applications.

The property of electronic optical band gap energy can be well exploited in the fields of terahertz generation and electro optical device applications [31] [32] [33]. The absorption coefficient $\alpha$ can be determined from the transmission spectrum based on the relation,

$$
\alpha=\frac{2.303 \log \left(\frac{1}{T}\right)}{t}
$$

where, $\alpha$ is the absorption coefficient, $T$ is the transmittance and $t$ is the thickness of the crystal. The band gap was estimated from the transmission spectrum and the optical absorption coefficient $\alpha$ near the absorption edge was calculated using the relation,

$$
h \gamma \alpha=A\left(h \gamma-E_{g}\right)^{1 / 2}
$$

In this equation $A$ is a constant, $E_{g}$ is electronic band gap energy, $h$ is plank's constant, $\gamma$ is frequency of the incident photon. Hence the optical band gap energy can be calculated from the Tau's plot between $(h \gamma \alpha)^{2}$ and the photon 


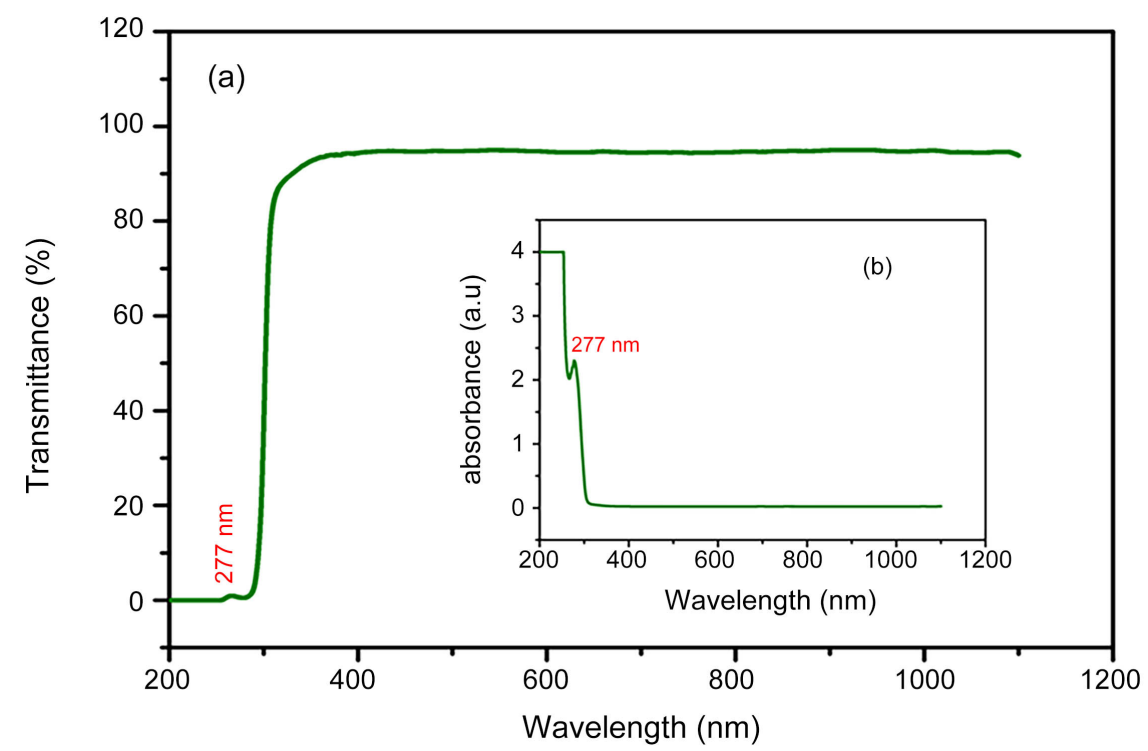

Figure 4. (a). UV-Vis transmission and (b) absorption spectrum of SAPB single crystal.

energy $h \gamma(\mathrm{eV})$ as exhibited in Figure 5. Then, by extrapolating the linear portion of Tauc's plot to the axis $\alpha h \gamma=0$, the value for optical band gap can be obtained [34] [35] [36] [37]. The band gap of SAPB crystal estimated by extrapolation of the linear part of the graph is $4 \mathrm{eV}$. The band gap and transmittance in the entire visible region enables the crystal suitable for optoelectronic and photonic applications.

\subsection{Fluorescence Study}

Fluorescence study is a prominent instrument to decide the actual properties of a material at the molecular level, including crystallinity and purity. The fluorescence spectrum was interpreted for SAPB crystal using Varian Cary Eclipse Fluorescence Spectrometer at room temperature. The fluorescence discharge range was recorded in the scope of 500 to $850 \mathrm{~nm}$, shown in Figure 6. The SAPB crystal showed a strong yellow emission peak at $574 \mathrm{~nm}$ and the red emission at 631 $\mathrm{nm}$ are identified [38] [39] [40]. A higher power ratio in the luminescence spectrum is an evidence of better purity, crystallinity, and structural perfection of the SAPB crystal; hence this semiorganic molecule with promising fluorescence emission can be exploited for NLO applications.

\subsection{Vickers Microhardness Analysis}

The mechanical properties of the crystal are very important for the practical device fabrication. The transparent SAPB single crystals free from cracks, with flat and smooth faces are chosen for static indentation Vickers microhardness test. In the present study, the applied loads $(P)$ are $25 \mathrm{~g}, 50 \mathrm{~g}$ and $100 \mathrm{~g}$ respectively and indentation time is fixed $10 \mathrm{~s}$ for all indentations. For each load, the average of two diagonal lengths ( $d$ ) of the indentation mark was measured with the help of calibrated micrometer attached to a metallographic microscope. The Vickers 


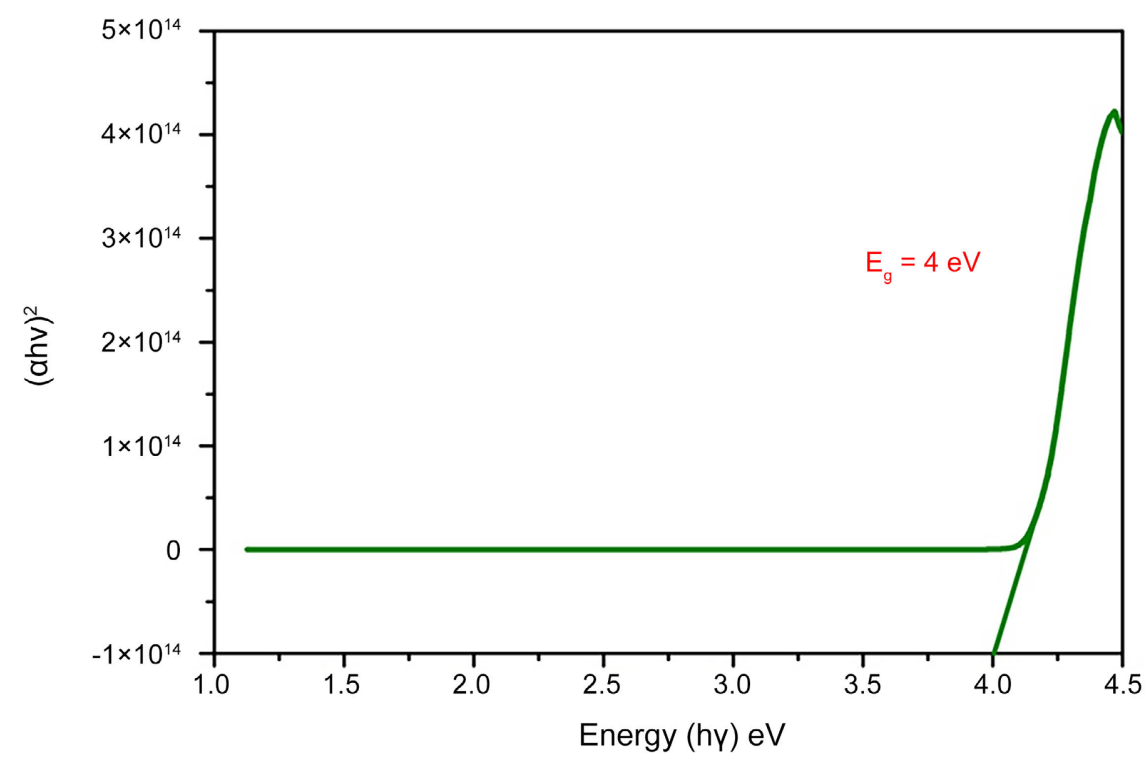

Figure 5. Tauc's plot of SAPB.

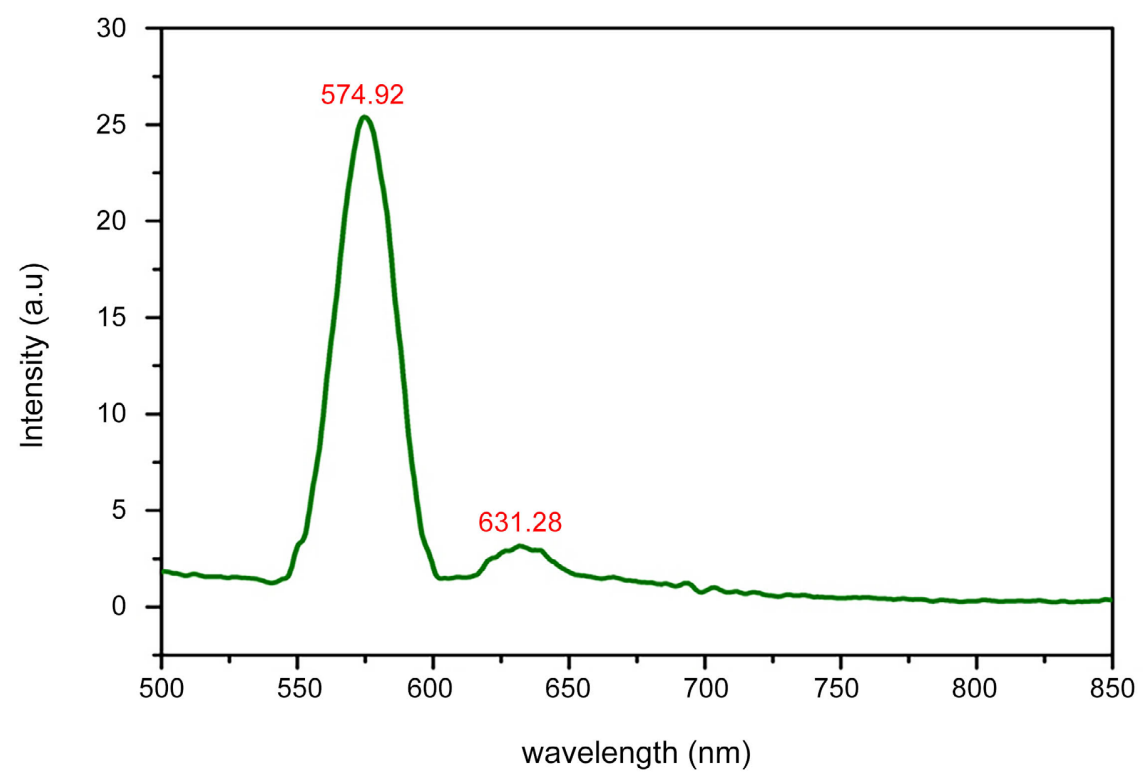

Figure 6. Fluorescence study of SAPB crystal.

hardness number $\left(H_{V}\right)$ value was calculated from the formula, $H_{V}=1.8544\left(P / d^{2}\right)$ $\mathrm{Kg} / \mathrm{mm}^{2}$ where $d$ is the diagonal length of the impression in $\mathrm{mm}$ and $P$ is the applied loads in $\mathrm{Kg}$. As a function of applied load $(P)$, the change in microhardness value $H_{v}$ and the plot of $\log d$ vs. $\log P$ are shown in Figure 7 and Figure 8 respectively. Further, the microhardness value increase of load and it is in agreement with the reverse indentation size effect [41] [42]. For above $100 \mathrm{~g}$ of applied load, micro cracks were observed due to the release of internal stress generated because of indentation process. The " $n$ " vale was calculated by using Mayer's law given by following relation $P=A d^{n}$ where $A$ is constant value and $n$ is the work hardening co-efficient. The Mayer's graph is shown in Figure 8. The 


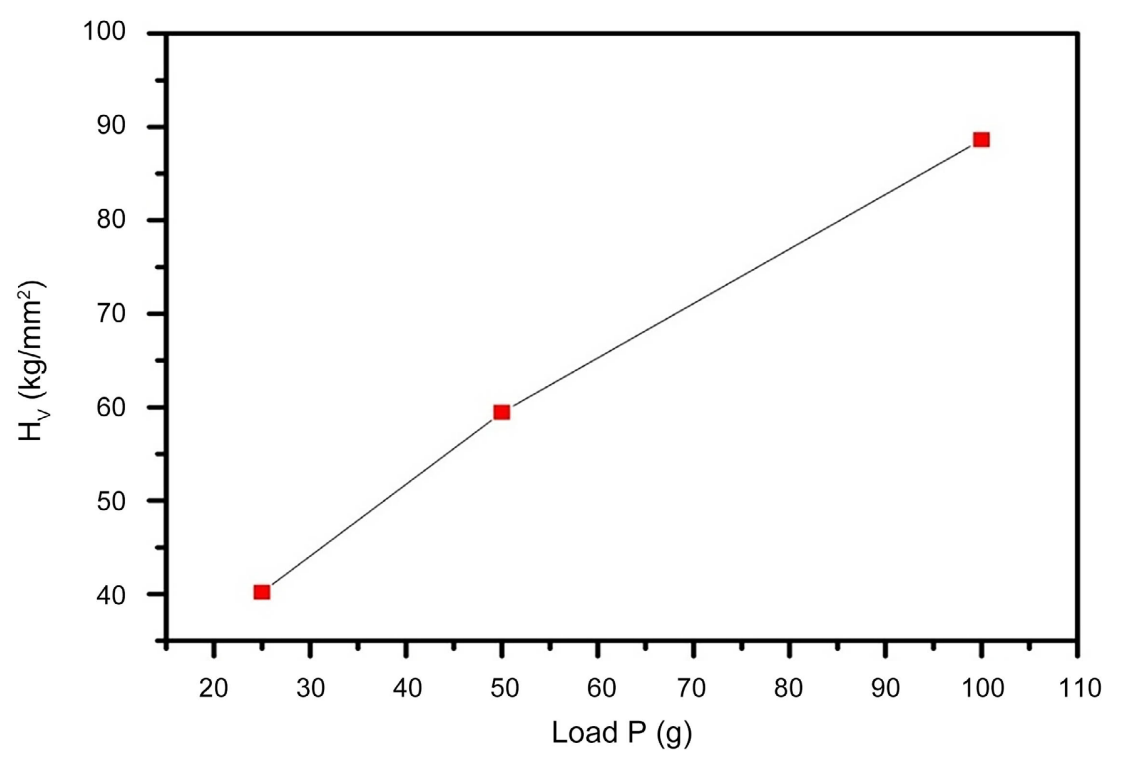

Figure 7. Variation of $H_{V}$ versus load $P$.

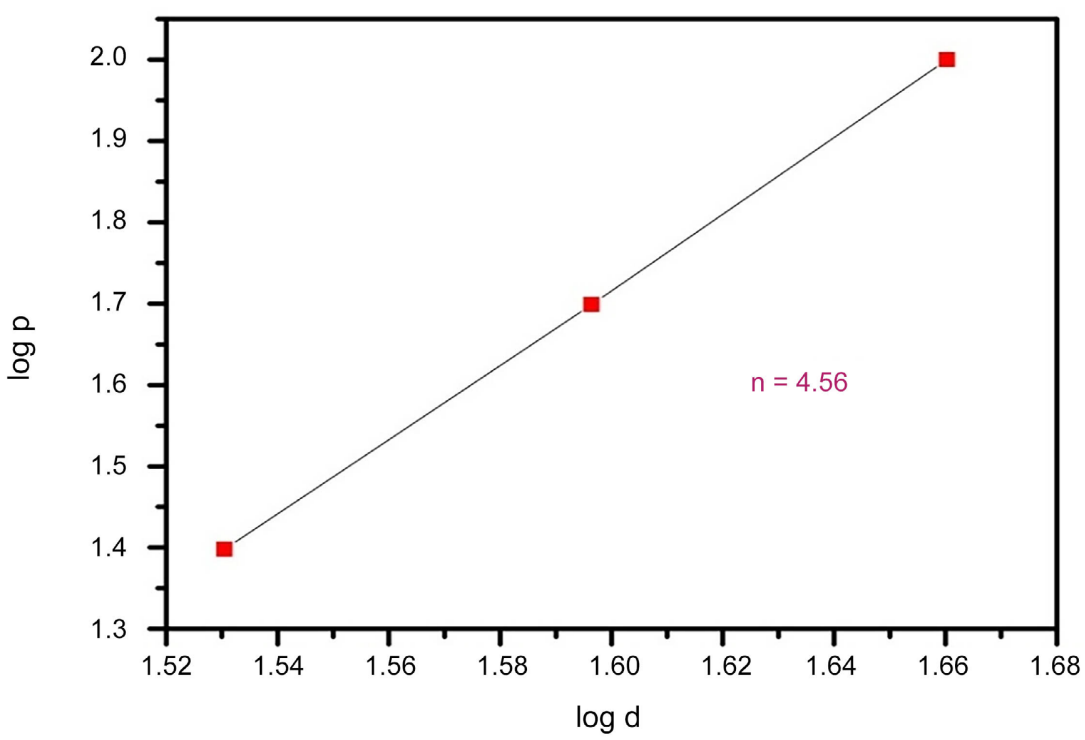

Figure 8. Graph between $\log P$ versus $\log d$.

work hardening co-efficient ( $n$ ) of the SAPB crystal has been determined from the slope of the straight line and it is found to be 4.56. According to Onitsch and Hanneman the value of $n$ comes out to be $1-1.6$ for hard materials and more than 1.6 for soft nature category. Hence, that grown SAPB single crystal belongs to the soft material category.

\subsection{Second Harmonic Generation Efficiency Test}

The non-linear optical activity of the grown crystal is investigated through the Kurtz-Perry powder method by performing out the SHG test. The frequency conversion effectiveness of material emphatically blends with the impurities, cationic deformities, and the intermolecular charge transfer interaction within the 
crystal lattice [43] [44]. The crystals are ground to powder and packed in a capillary tube and were exposed to the continuous wave mode bolted Q-switched Nd-YAG laser of fundamental wavelength $1064 \mathrm{~nm}$ delivering $10 \mathrm{~ns}$ laser pulses following a repetition rate of $10 \mathrm{~Hz}$. The SHG behavior in these crystals was confirmed from the outflow of exceptional green radiation $(\lambda=532 \mathrm{~nm})$ by the sample. The determined SHG efficiency of SAPB of crystal as 2 times superior to KDP crystal.

\section{Conclusion}

Optically good quality single crystal of benzophenone doped sodium acid phthalate crystals has been grown by slow solution evaporation technique. The sharp distinct Bragg's peaks confirm the crystalline nature of the synthesized material. The crystallographic contemplates were completed out utilizing the Single XRD structural pattern and found that SAPB crystal belongs to monoclinic system with $\mathrm{B} 2 \mathrm{cb}$ as the non-centrosymmetric space group. The functional groups of the SAPB crystal have been detected by FTIR spectroscopy. UV-Vis spectrum confirms the wide optical transparency of the compound of wavelength with optical band gap $4 \mathrm{eV}$, which guarantees the ability of the material to create higher harmonic frequencies in non-linear optical applications. The luminescence property of the material was examined from Fluorescence studies with an emission peak at $574.72 \mathrm{~nm}$ exhibits its utility in yellow light emitting diodes. The value of Mayer's index was calculated as 4.56 , which suggests that SAPB belongs to soft material category. The nonlinear optical test revealed that SAPB determined SHG efficiency of 2 times that of KDP. The above investigations conclude that the benzophenone doped sodium acid phthalate crystal could be advantageous for applications of various frontier technological photonic devices utilized in optical limiting systems, night vision sensors, optical logic switching, signaling, and modulating system.

\section{Acknowledgements}

The authors are grateful to the SAIF, IIT madras, for providing facilities such as single crystal XRD and Archbishop Casimir Instrumentation center, St. Joseph's college Tiruchy for providing the UV, Fluorescence analysis. Dr. P.K. Das Laboratory, IISc, Bangalore, is acknowledged for rendering SHG analysis.

\section{Conflicts of Interest}

The authors declare that they have no known competing financial interests or personal relationships that could have appeared to influence the work reported in this paper.

\section{References}

[1] Nirmala Sri Devi, S., Arunkumar, R. and Girija, E.K. (2015) Investigations on the Effect of Transition Metals on the Growth and Characterization of Sodium Acid 
Phthalate Single Crystals. Optik, 127, 2419-2423.

https://doi.org/10.1016/j.ijleo.2015.11.176

[2] Alsaad, A.M., Al-Bataineh, Q.M., Ahmab, A. and Zaid Albataineh, A.T. (2020) Optical Band Gap and Refractive Index Dispersion Parameters of Boron-Doped ZnO Thin Films: A Novel Derived Mathematical Model from the Experimental Transmission Spectra. Optik, 211, Article ID: 164641.

https://doi.org/10.1016/j.ijleo.2020.164641

[3] Sethuraman, K., Ramesh Babu, R., Vijayan, N., Gopikrishnan, R. and Ramasamy, P. (2006) Synthesis, Growth of Organic Nonlinear Optical: Semicarbazone of 2-Amino-5-chloro-benzophenone (S2A5CB) and Its Characterization. Journal of Crystal Growth, 290, 593-543. https://doi.org/10.1016/j.jcrysgro.2006.01.038

[4] Saravanan, D., Senthil, V.P., Gokulraj, S., Sudalairaja, G., Anbalagan, G. and Ramesh Kumar, G. (2017) Growth of Bulk Sodium Acid Phthalate (NaAP) Crystals for High Frequency Laser Generation in Nonlinear Optical Applications. Materials To day Proceedings, 4, 758-762. https://doi.org/10.1016/j.matpr.2017.01.083

[5] Marudhu, G., Krishnan, S. and Vijayaraghavan, G.V. (2014) Optical, Theoretical and Mechanical Studies on Sodium Acid Phthalate Crystals. Optik, 125, 2417-2421. https://doi.org/10.1016/j.ijleo.2014.01.008

[6] Vivek, P., Rekha, M., Ananth Steephen, Jauhar, R.O.M.U., Saravanakumar, G., Suvitha, A., Karunagaran, N. and Kowsalya, M. (2021) Nonlinear Optical Crystalline Nature Bis(2,6-diaminopyridinium) Hydrogen Phthalate Nitrate (APPN): Development and Its Phase Matching Nature. Results in Optics, 3, Article ID: 100075. https://doi.org/10.1016/j.rio.2021.100075

[7] Anandaraj, L., Jothi, L., John Sundaram, S. and Terence, N. (2021) Blue Emissive Properties of Pure and Sodium Acid Phthalate Doped Hippuric Acid Crystal for NLO Application. Materials Today Proceedings, 36, 514-519. https://doi.org/10.1016/j.matpr.2020.05.307

[8] Usharani, S., Judes, J., Natarjan, V., Arivanadhan, M., Anandan, P., Dmitry, A. and Vorontsov, M.O. (2020) The Effect of Mixed Solvents on Solute-Solvent Interactions and Bulk Growth of 3,4-Diamino Benzophenone: A Novel Benzophenone Derivative for Nlo Application. Optical Materials, 100, Article ID: 109603. https://doi.org/10.1016/j.optmat.2019.109603

[9] Maradu, G., Krishnan, S. and Vijayaraghavan, G.V. (2014) Optical, Theoretical and Mechanical Studies on Sodium Acid Phthalate Crystal. Optik, 125, 2417-2421. https://doi.org/10.1016/j.ijleo.2014.01.008

[10] Nandhini, S., Muniyappan, S., Ramar, V., Balasubramanian, K. and Murugakoothan, P. (2019) Quantum Chemical Analysis on Supramolecular Assemblies of Guanidinium Tetrafluoroborate (GFB) Crystal Structure: Emission and NLO Behavior. Journal of Molecular Structure, 1198, Article ID: 126859.

https://doi.org/10.1016/j.molstruc.2019.07.106

[11] Suvitha, A. and Murugakoothan, P. (2012) Synthesis, and Growth, Structural, Spectroscopic and Optical Studies of a Semiorganic NLO Crystal: Zinc Guanidinium Phosphate. Spectrochimica Acta Part A: Molecular and Biomolecular Spectroscopy, 86, 266-270. https://doi.org/10.1016/j.saa.2011.10.034

[12] Manimekalai, R., Antony Joseph, A. and Ramachandra Raja, C. (2014) Crystal Growth and Spectroscopic Characterization of Aloevera Amino Acid Added Lithium Sulfate Monohydrate: A Non-Linear Optical Crystal. Spectrochimica Acta Part A: Molecular and Biomolecular Spectroscopy, 122, 232-237.

https://doi.org/10.1016/j.saa.2013.11.085 
[13] Suresh, S. (2014) Growth, Optical, Mechanical, Dielectric and Theoretical Properties of Picolinium Maleate NLO Single Crystal. Optik, 125, 2826-2829. https://doi.org/10.1016/j.ijleo.2013.11.065

[14] Senthil Pandian, M. and Ramasamy, P. (2012)Sodium Sulfanilate Dehydrate (SSDH) Single Crystals Growth by Conventional Slow Evaporation and Sankaranarayanan Ramasamy (SR) Method and Its Comparative Characterization Analysis. Materials Chemistry and Physics, 132, 1019-1028.

https://doi.org/10.1016/j.matchemphys.2011.12.057

[15] Senthil, A., Ramasamy, P. and Verma, S. (2011) Investigations on the SR Method Growth, Etching Birefringence, Laser Damage Threshold and Dielectric Charaterization of Sodium Acid Phthalate Single Crystal. Journal of Crystal Growth, 318, 757-761. https://doi.org/10.1016/j.jcrysgro.2010.11.115

[16] Thomas Joseph Prakash, J., Vijayan, N. and Kumararaaman, S. (2008) Growth and Characterization Studies on Glycine Barium Dichloride Single Crystal for NLO Applications. Spectrochimica Acta Part A: Molecular and Biomolecular Spectroscopy, 71, 1250-1252. https://doi.org/10.1016/j.saa.2008.03.022

[17] Thilagavathy, S.R., Rajesh, P., Ramasamy, P. and Ambujam, K. (2014) Growth and Characterization of Pure and Doped KHP NLO Single Crystals. Spectrochimica Acta Part A: Molecular and Biomolecular Spectroscopy, 127, 248-255. https://doi.org/10.1016/j.saa.2014.01.137

[18] Bairava Ganesh, R., Kannan, V., Meera, K., Rajesh, N.P. and Ramasamy, P. (2005) Synthesis, Growth and Chaeacterization of a New Non Linear Optical Crystal Sodium Acid Phthalate. Journal of Crystal Growth, 282, 429-433.

https://doi.org/10.1016/j.jcrysgro.2005.05.034

[19] Ruby Nirmala, L. and Prakash, J. (2013) Effect of L-Valine on the Growth and Characterization of Sodium Acid Phthalate (SAP) Single Crystals. Spectrochimica Acta Part A: Molecular and Biomolecular Spectroscopy, 10, 425-429. https://doi.org/10.1016/j.saa.2013.03.070

[20] Goel, N. and Kumar, B. (2012) Remarkable Enhancement in Optical Properties of Sodium Hydrogen Phthalate Hemihydrate Crystals Due to $\mathrm{Fe}^{3+}$ Doping. Journal of Crystal Growth, 361, 44-50. https://doi.org/10.1016/j.jcrysgro.2012.08.044

[21] Ambrose Rajkumar, M., Stanly John Xavier, S., Anbarasu, S. and Prem Anand, D. (2016) Growth and Characterization Studies of an Efficient Semiorganic NLO Single Crystal: 2-Amino 5-nitropyridinium Dihydrogen Phosphate (2A5NPDP) by Sankaranarayanan-Ramasamy Method. Optik, 127, 2187-2192.

https://doi.org/10.1016/j.ijleo.2015.10.239

[22] Manivannan, M., Martin Britto Dhas, S.A. and Jose, M. (2016) Photoacoustic and Dielectric Spectroscopic Studies of 4-Dimethylamino-n-methyl-4-stilbazolium Tosylate Single Crystal: An Efficient Terahertz Emitter. Journal of Crystal Growth, 455, 161-167. https://doi.org/10.1016/j.jcrysgro.2016.09.053

[23] Saravanan, D., Senthil, V.P., Gokul raj, S., Sudalai Raj, G., Anbalagan, G. and Ramesh Kumar, G. (2017) Growth of Bulk Sodium Acid Phthalate (NaAP) Crystals for High Frequency Laser Generation in Nonlinear Optical Applications. Materials Today Proceedings, 4, 758-762. https://doi.org/10.1016/j.matpr.2017.01.083

[24] Senthil, A. and Ramasamy, P. (2009) Unidirectional Growth of $<001>$ Sodium Acid Phthalate Single Crystal by Sankaranarayanan-Ramasamy (SR) Method. Journal of Crystal Growth, 311, 4720-4724. https://doi.org/10.1016/j.jcrysgro.2009.09.014

[25] Vediyappan, S., Arumugam, R., Pichan, K., Kasthuri, R., Pandian, S. and Perumal, R. (2017) Crystal Growth and Characterization of Semi-Organic 2-Amino-5-nitro- 
pyridinium Bromide (2A5NPBr) Single Crystals for Third-Order Nonlinear Optical (NLO) Application. Applied Physics A, 123, 780-795.

https://doi.org/10.1007/s00339-017-1394-3

[26] Rajkumar, R. and Praveen Kumar, P. (2019) Structure, Crystal Growth and Characterization of Piperazinium Bis(4-nitrobenzoate) Dihydrate Crystal for Nonlinear Optics and Optical Limiting Applications. Journal of Molecular Structure, 1179, Article ID: 108117. https://doi.org/10.1016/j.molstruc.2018.10.085

[27] Nageshwari, M., Jayaprakash, P., Rathika Thaya Kumari, C., Vinitha, G. and Lydia Caroline, M. (2017) Growth, Spectral, Linear and Nonlinear Optical Characteristics of an Efficient Semiorganic Acentric Crystal: L-Valine Chloride. Physica B: Condensed Matter, 511, 1-9. https://doi.org/10.1016/j.physb.2017.01.027

[28] Rajagopalan, N.R., Krishnamoorthy, P. and Jayamoorthy, K. (2017) A Strategic Approach to Physico-Chemical Analysis of Bis(thiourea) Lead Chloride-A Reliable Semi-Organic Nonlinear Optical Crystal. Optics and Laser Technology, 89, 6-15. https://doi.org/10.1016/j.optlastec.2016.10.001

[29] Mao, C.J., Zhou, G.G., Sun, X., et al. (2020) Optical and Thermal Properties of 1-Tartaric Doped KDP Crystals. Optik, 203, Article ID: 163967.

https://doi.org/10.1016/j.ijleo.2019.163967

[30] Prakash, M., Geetha, D. and Ladiya caroline, M. (2011) Crystal Growth and Characterization of L-phenylalaninium Trichloroacetate, a New Organic Nonlinear Optical Material. Physica B Condensed Matter, 406, 2621-2625. https://doi.org/10.1016/j.physb.2011.04.002

[31] Gayathri, K., Krishnan, P., Rajkumar, P.R. and Anbalagana, G. (2014) Growth, Optical, Thermal and Mechanical Characterization of an Organic Crystal: Brucinium 5-Sulfosalicylate Trihydrate. Bulletin of Materials Science, 37, 1589-1595. https://doi.org/10.1007/s12034-014-0721-y

[32] Mande, P., et al. (2017) NLO Properties of 1,4-naphthoquinone, Juglone and Lawsone by DFT and Z-Scan Technique-A Detailed Study. Optical Materials, 72, 549-558. https://doi.org/10.1016/j.optmat.2017.06.058

[33] Esthaku Peter, M. and Ramasamy, P. (2010) Synthesis, Growth and Characterization of a Novel Semiorganic NLO Crystal: Triglycine Calcium Dibromide. Journal of Crystal Growth, 312, 1952-1956. https://doi.org/10.1016/j.jcrysgro.2010.03.015

[34] John sundaram, S., Jerald, Ramaclus, V., Paneerselvam, M., Jocob, M., Priya antony, and Anandaraj, L. (2020) Crystal Growth and Characterization of 4-[4-(4-dimethylamino-phenyl)buta-1,3-dienyl]-1-methyl Pyridinium Iodide for Higher Order Non-Linear Applications. Optical and Laser Technology, 121, Article ID: 105831. https://doi.org/10.1016/j.optlastec.2019.105831

[35] Madeswaran, P. and Chandrasekaran, J. (2011) Synthesis, Growth and Characterization of L-Valine Cadmium Chloride Monohydrate-A Novel Semiorganic Nonlinear Optical Crystal. Optik, 122, 1128-1131.

https://doi.org/10.1016/j.ijleo.2010.07.006

[36] Devi, S.N.S., Kumar, R.A. and Girija, E.K. (2016) Investigations on the Effect of Transition Metals on the Growth and Characterization of Sodium Acid Phthalate Single Crystals. Optik, 127, 2419-2423. https://doi.org/10.1016/j.ijleo.2015.11.176

[37] Raja, M., Raj Muhamed, R., Muthu, S. and Suresh, M. (2017) Synthesis, Spectroscopic (FT-IR, FT-Raman, NMR, UV-Visible), NLO, NBO, HOMO-LUMO, Fukui Function and Molecular Docking Study of (E)-1-(5-bromo-2-hydroxybenzylidene) Semicarbazide. Journal of Molecular Structure, 1141, 284-298.

https://doi.org/10.1016/j.molstruc.2017.03.117 
[38] Govindarsu, K. and Kavitha, E. (2014) Molecular Structure, Vibrational Spectra, NBO, UV and First Order Hyperpolarizability, Analysis of 4-Chloro-DL-phenylalanine by Density Functional Theory. Spectrochimica Acta Part A: Molecular and Biomolecular Spectroscopy, 133, 799-810. https://doi.org/10.1016/j.saa.2014.06.019

[39] Karthiga Devi, P., Venkatachalam, K. and Poonkotthai, M. (2016) Spectroscopic, Optical, Thermal, Antimicrobial and Density Functional Theory Studies of 4-Aminopyridinium 4-Hydroxy Benzoate Hydrate Crystal. Journal of Molecular Structure, 1119, 462-471. https://doi.org/10.1016/j.molstruc.2016.05.001

[40] Chaouachi, S., Elleuch, S., et al. (2016) Experimental (FTIR, Raman, Uvevisible and $\mathrm{PL}$ ) and Theoretical (DFT and TDDFT) Studies on Bis(8-hydroxyquinolinium) Tetrachlorocobalate (II) Compound. Journal of Molecular Structure, 1125, 149-161. https://doi.org/10.1016/j.molstruc.2016.06.050

[41] Karuppasamy, P., Pandian, M. and Ramasamy, P. (2017) Crystal Growth and Characterization of Third Order Nonlinear Optical Piperazinium Bis(4-hydroxybenzenesulphonate)(P4HBS) Single Crystal. Journal of Crystal Growth, 473, 39-54. https://doi.org/10.1016/j.jcrysgro.2017.05.018

[42] Ishikawa, H. and Shikai, N. (2006) Critical Load for Median Crack Initiation in Vickers Indentation of Glasses. Journal of the American Ceramic Society, 65, c124-c127. https://doi.org/10.1111/j.1151-2916.1982.tb10496.x

[43] Mohana, J., Ahila, G., Bharathi, D.M. and Anbalagan, G. (2016) Growth, Spectral, Optical, Thermal, and Mechanical Behaviour of an Organic Single Crystal: Quinolinium 2-Carboxy 6-Nitrophthalate Monohydrate. Journal of Crystal Growth, 450, 181-189. https://doi.org/10.1016/j.jcrysgro.2016.06.044

[44] Smith, R.A. (1975) The Crystal Structures of a Series of Salts of Phthalic Acid. II. The Crystal Structure of Sodium Acid Phthalate Hemihydrate. Acta Crystallographica Section B, 31, 2345. https://doi.org/10.1107/S0567740875007546 